\title{
A Study on the Difficulties for College Students in Their Chunk Learning Process
}

\author{
Ma Songqing \\ Zhonghuan Information College Tianjin University of Technology, Tianjin, China, 300380 \\ marchea@126.com
}

Keywords: chunk learning; lexical chunks; Lexical Approach

\begin{abstract}
This paper is a study on the application of lexical-chunk theory proposed by Lewis in college English teaching, aiming at investigating what specific difficulties learners probably meet in chunk learning process by means of translation tests. Research results indicate that in the chunk learning process, students have indeed encountered various difficulties, such as syntactical functions, culture comprehension, preposition usage and verb-noun chunk collocation.
\end{abstract}

\section{Introduction}

Lexical Approach is mainly dedicated to students' learning and mastering of lexis, or words and word combinations. It is based on the idea that the essential part of language acquisition is the ability to comprehend and produce lexical chunks as an unanalyzed whole. These chunks serve as the basis on which students perceive language materials, which used to be traditionally analyzed with grammar (Lewis, 1993:95). It is blessed with many advantages for language teaching. For example, it enables learners to use the ready-made expressions, which could hardly be possible to be creatively produced, because these chunks are mostly subject to linguistic analysis and could be further segmented according to regular linguistic rules; the use of the Lexical Approach in language teaching seems to avoid the pitfalls warned by Widdowson "too heavy reliance on either structural model of competence, or communicative model of appropriate use" (Widdowson,1989:247); the common route for acquiring a language is usually regarded as from memorized routine to creative use of language (Ellis, 1999).

It has to be admitted that both college non-English majors' English proficiency and methodology in English teaching have been considerably improved in China. However, it is also widely recognized that though vocabulary learning takes a large amount of time and energy of both students and teachers, the ultimate learning effect is far from satisfactory. For quite a number of college students, they may encounter specific difficulties.

\section{Research Methodology}

The subjects in this research involve 78 freshmen of non-English major in Zhonghuan Information College, Tianjin University of Technology. All the subjects are native speakers of Chinese. They were admitted into this college in the fall of 2013. Two mini-tests of chunk learning are conducted both in the middle and at the end of the study. The tests all in all are made up of 25 Chinese sentences for translation, which intend to test subjects' knowledge of chunks and their productive ability of chunks on a regular basis.

\section{Results and Discussions}

From the data account of students' performance, it can been seen that a number of typical chunks have indeed been acquired by the students, such as enlarge one's vocabulary, by no means, at random, in part and come up with, etc.. However, the general result is still far from desirable when we take into account both the quantity and quality of chunks produced. While some chunks have been recognized by most of the students, some others have been misused, such as (be) well organized, except for, win the first prize and with a wide range of, etc.. All the data reveals that in spite of the help of Lexical Approach, our English learners still encounter certain obstacles in chunk leaning. The following part is devoted to the discussion of the specific difficulties.

\subsection{Difficulty in syntactical functions}

As one would expect, even at an advanced level of proficiency, a variety of grammatical errors can still be found in students' performance. Most of them are very minor errors regarding to 
syntactical functions, which are quite many in our tests. Some typical ones are chosen as below as examples:

(1) * On her birthday, she seemed satisfy to receive a bunch of flowers from her boyfriend.

(2) * According to our English teacher, we should have our own learn strategies.

(3) * After reading the letter, the old man felt disappoint/ disappointedly and tore it into little bits.

(4) * According to the doctor introduce, this medicine can be used to relive painful.

(5) * He has always wanted everything to be good organized.

(6) * Doing exercise every morning has made a good basic for my physical health.

(7) * He felt embarrass when giving a public speech in class meeting.

(8) * He exclaimed in exciting when he knew he had won the first prize.

As can be seen, all of the examples involve errors in forming grammatical collocations, especially the misusage of syntactical functions in some chunks. For instance, the right form in case (1) should be seem satisfied and case (2) learning strategies. This phenomenon is indeed common among Chinese learners. Many of them do not lay a good foundation in grammar learning when they are in middle school or high school and lack the ability of recognizing and using words in right form. In the process of chunk learning, students are gradually accustomed to remember the chunk as a whole like an individual word. However, the inner grammatical relation among the individual words of a chunk is equally important. Many students with relatively poor grammar have difficulty in this aspect.

\subsection{Difficulty in culture comprehension}

Vocabulary, as the most active element in language, often embodies social life and reflects social culture such as people's way of life, mode of thinking, value judgment and historical background. However, duo to the historical, geographic and political reasons, there is a big difference between Chinese culture and western culture. This kind of cultural difference is often reflected on chunk meaning, which in turn makes cross-cultural communication more difficult.

For example, blue, denoting a color, is a favorable word to Chinese, such as the blue sky, the blue sea. While in western culture, blue does not associate with happiness and imagination, but gloom and depression, for instance, "He has been in blue mood." means that he has been in a gloomy or sad mood.

In the translation tests several culture-related chunks are involved. For example, in sentence 17 the answer to “幸运儿” would be lucky dog. Dog in Chinese is often associated with some derogatory connotation, such as 走狗、狗官, etc.. In most cases of English language, dog is neural in its connotation. It is all right to refer to certain people as big dog, top dog, lucky dog, sly dog, etc.. A lucky dog just means a person who is lucky and is widely used in spoken English. In sentence 18 “最大的份额” is favorably translated as lion’s share. In western culture, lion is regarded as the king of forest and always possess the largest and best part of everything. Therefore, lion's share means “the largest share” or "the best share”. In sentence 25 “随大流” and follow suits would be equivalent pair. In the west, there is no earlier meaning for follow suit than the one from card games, "to play a same suit as the leading card". Hence, follow suit means to do the same thing as somebody or something else.

From students' translation performance, it can be seen that only $26 \%$ (sentence 17), $13 \%$ (sentence 18) and 14\% (sentence 25) separately of the learners can make out the suitable answer. So in terms of chunk learning, a majority of students so far haven't acquired this kind of culture-related chunks and found it hard to gain a good command of these special items.

\subsection{Difficulty in preposition-chunk usage}

The usage of various prepositions is a quite common phenomenon in English language. And suitable and wonderful usage of a preposition could contribute to the quality, fluency and beauty of 
a sentence. For instance,

(a) The school has a long history of more than 100 years and is quite famous.

(b) The school is quite famous with a long history of more than 100 years.

Obviously, with the proper use of preposition with sentence (b) seems more idiomatic and fluent than sentence (a). While in Chinese the preposition is not as active or frequently-used as other grammatical units, which is one of the characteristics in Chinese compared with English. Hence, this difference in preposition usage makes it more difficult and complicated for Chinese learners. In fact, many students often ignore the employment of different preposition expressions, which may lead to high rate of simple sentences instead of idiomatic preposition chunks. Some detailed examples in translation tests are illustrated as below:

In sentence 1, the target chunk is on a regular basis equivalent to “经常”. The whole sentence translated as reference is Listening to English news on a regular basis will not only improve your ear but also enlarge your vocabulary. According to the results, only $26 \%$ of subjects can recognize and use the target chunk, and $68 \%$ of the students just employ single words, such as often, regularly, frequently, to modify the sentence.

In sentence 2, the target chunk round the corner refers to “即将到来”. The favorable translation should be The final examination is round the corner. 44\% students can put this chunk into practical translation practice, but most of the left $56 \%$ mostly make the sentence without preposition chunks, such as The final examination is coming/will come.

In sentence 24, the whole sentence is referred as She is a lively girl with a wide range of interests with with a wide range of as the target chunk. Data show that only $27 \%$ subjects can make it by themselves, the majority of them often use some simple verb expressions, such as she is a lively girl and has a lot of interests.

From the examples, it can be seen that although a minor of learners can use preposition chunks in right place, the majority are not used to use them and have difficulty in active awareness of function of preposition chunks.

\subsection{Difficulty in verb-noun collocation}

Verbs play an important and active role in English language. For the verb chunks, especially verb collocations, it is required to employ suitable verbs to modify a noun, otherwise there would be the occurrence of overlapping or blending. For example, “承担责任” may be translated as perform the duty or take the responsibility. It is quite common and easy for Chinese learners to translate as perform the responsibility, which is the result of blending the two idiomatic chunks.

In the tests, similar problems are found as the following:

In sentence 1, the target chunk is improve one's ear. $31 \%$ of the subjects have equated improve and develop or increase in meaning, hence believing that they can be used interchangeably.

In sentence $8,33 \%$ of the students take finish/ complete one's dream as an acceptable chunk though $49 \%$ of them can make out the suitable expressions as accomplish one's old dream or realize one old dream. By using finish or complete instead of accomplish or realize, many of them have done this according Chinese thinking method, which is not acceptable in English.

In sentence 10, more than $23 \%$ of the subjects are confused with keep up with and come up with because they are alike in appearance.

And in sentence 25, the best target chunk may be draw/ reach/ come to a conclusion. Students may choose any verb from the above. Actually $44 \%$ of the learners choose get or make to modify the noun, influenced by the Chinese thinking method.

On the whole, Chinese learners are not proficient in collocating right verbs with nouns and have difficulty in overcoming such problems as blending or the obstacle of Chinese thinking method.

\section{Conclusion}

In the chunk learning process, students have indeed acquired a number of chunks. However, students still meet obstacles in various aspects, such as syntactical functions, culture comprehension, 
preposition usage and verb usage.

As the present study shows, even advances learners have considerable difficulties in the recognition and production of lexical chunks. A re-recognition of the phenomenon of the chunks is hence of vital importance to both theoretical researches and pedagogical practices.

Teachers have the responsibility to help students out of the difficulties in chunk learning. In teaching process, it is suitable to combine chunks with grammar rather than make them clearly cut due to some learner's lack of grammar rules. Teachers are expected to provide more information about culture of different languages, which is beneficial to the understanding of cultural chunks. What's more, teachers should pay more attention to the explanation of structural similarities and differences in order to better use the preposition chunks. Verb-noun collocation is really a difficulty for Chinese learners when choosing the right verbs. Both teachers and students should accumulate idiomatic expressions and make comparison as much as possible.

\section{References}

[1] Ellis R. the Study of Second Language Acquisition [M]. Shanghai: Shanghai Foreign Language Education Press, 1999

[2] Lewis Michael. The Lexical Approach: The state of ELT and a way forward. Hove England: Language Teaching Publications, 1993

[3] Lewis Michael. Implementing the Lexical Approach: Putting Theory into Practice. Hove England: Language Teaching Publications, 1997

[4] Oxford R. Language Learning Strategies: What Every Teacher Should Know. Newburry House, 1990

[5] Widdowson, H. G. Knowledge of language and ability for use [J]. Applied Linguistics 10: 1989. $128-137$ 